Received: 13 October 2017

Accepted: 1 November 2018

Published online: 07 December 2018

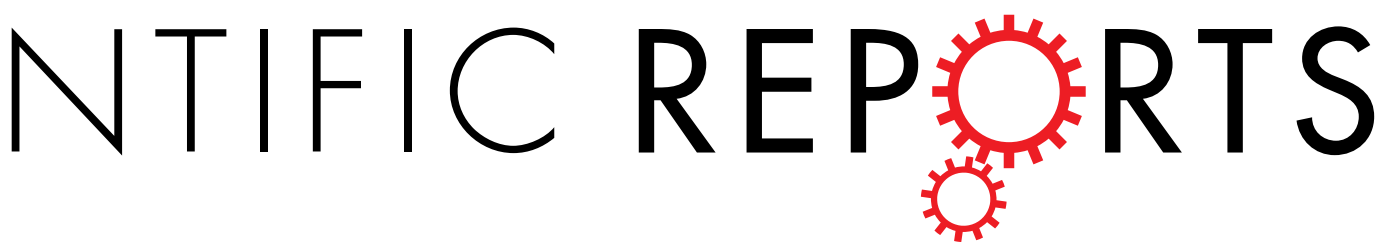

\title{
OPEN The expression of ecdysteroid UDP-glucosyltransferase enhances cocoon shell ratio by reducing ecdysteroid titre in last-instar larvae of silkworm, Bombyx mori
}

\author{
Guanwang Shen ${ }^{1,2}$, Jinxin $\mathrm{Wu}^{1}$, Yong Wang ${ }^{3}$, Hongling Liu ${ }^{1}$, Haiyan Zhang ${ }^{1}$, Sanyuan $\mathrm{Ma}^{1}$, \\ Chuyue Peng ${ }^{3}$, Ying $\operatorname{Lin}^{1,2}$ \& Oingyou Xia ${ }^{1,2}$
}

Ecdysteroid UDP glucosyltransferase (EGT) is a baculovirus-encoded protein which can hinder the normal molting of insects by inactivating 20-hydroxyecdysone (20E). Here we expressed EGT in the last-instar silkworm larvae using the GAL4/UAS system. Compared with the control, for the EGT overexpressed silkworm, the hemolymph $20 \mathrm{E}$ content was significantly decreased, the feeding and spinning periods of the last-instar silkworm larvae were extended, the cocoon shell ratio was significantly increased, and the transformation from silkworm larvae to pupa was blocked. Increasing EGT expression resulted in the decrease of $20 \mathrm{E}$ content in the hemolymph of silkworm larvae, treating the EGT overexpressed male silkworm with 20E decreased the larval weight and cocoon shell ratio, confirming that the increase in the availability of nutrients to the cocoon and an increase in the cocoon shell weight in the hybrid transgenic silkworms is because of the EGT-induced reduction in active $20 \mathrm{E}$ content. Furthermore, though the sericin and flavonoid contents were increased in the cocoon of the EGT overexpressing silkworm, the production of silk fibroin didn't change.

The silkworm (Bombyx mori) is a representative member of the order Lepidoptera. It has many advantages as a model organism, including availability of the complete genome sequence ${ }^{1,2}$, short life cycle, moderate size, and easy cultivation ${ }^{3-6}$. Thus, the silkworm has been widely used in molecular biology, physiology, developmental, toxicology, and medical studies. It has four distinct developmental stages including egg, larva, pupa, and moth ${ }^{7}$. Larvae undergo several rounds of moulting, during which nutrients taken up from mulberry leaves are used to construct the cocoon and prepare for pupal development and adult organ formation. The other three developmental stages mainly serve to allocate nutrients accumulated in larvae.

Pupae are the intermediate stage in the metamorphosis from larva to adult. The transition from late last-instar larva to early pupa is the most important period for nutrient distribution, as larvae stop feeding and start spinning a protective cocoon. The allocation of nutrients for this process is under strict genetic and hormonal control. Ecdysone (20-hydroxyecdysone, 20E) regulates the development of the pre-pupa ${ }^{8}$; the level is relatively low in the spinning stage and increases after spinning ${ }^{9,10}$. A high concentration of $20 \mathrm{E}$ in the haemolymph promotes the degradation of larval tissues including silk glands ${ }^{11-13}$, leading to pupation ${ }^{14,15}$. However, it is unclear whether $20 \mathrm{E}$ affects the absorption and utilization of nutrients in silk production. If so, modulating $20 \mathrm{E}$ levels could delay pupation and prolong the spinning stage, leading to increased silk production.

Ecdysteroid UDP glucosyltransferase (EGT) is a baculovirus-encoded protein ${ }^{16,17}$ that is secreted by Acanthopanax sp. heterozygous nucleopolyhedrovirus (NPV) and transfers UDP-glucoside to the hydroxyl group of carbon 22 in $20 \mathrm{E}$ to generate 20E 22- $\beta$-D-pyran glucoside ${ }^{18,19}$. The EGT secreted by the virus upon infection can inactivate $20 \mathrm{E}$ in the host, thereby hindering normal moulting and eclosion ${ }^{20-22}$. Transient expression of the EGT

${ }^{1}$ State Key Laboratory of Silkworm Genome Biology, Southwest University, Chongqing, 400716, China. ${ }^{2}$ Chongqing Engineering and Technology Research Center for Novel Silk Materials, Chongqing, 400716, China. ${ }^{3}$ College of Biotechnology in Southwest University, Chongqing, 400716, China. Correspondence and requests for materials should be addressed to Q.X. (email: xiaqy@swu.edu.cn) 

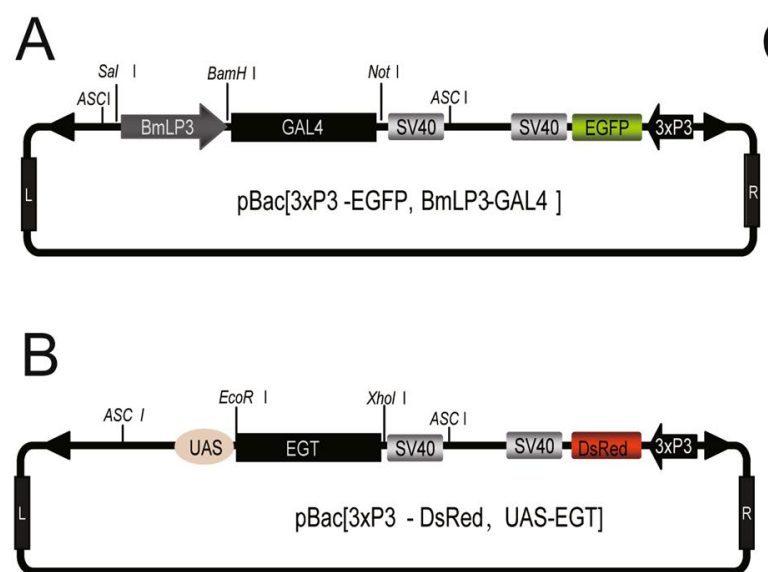

C
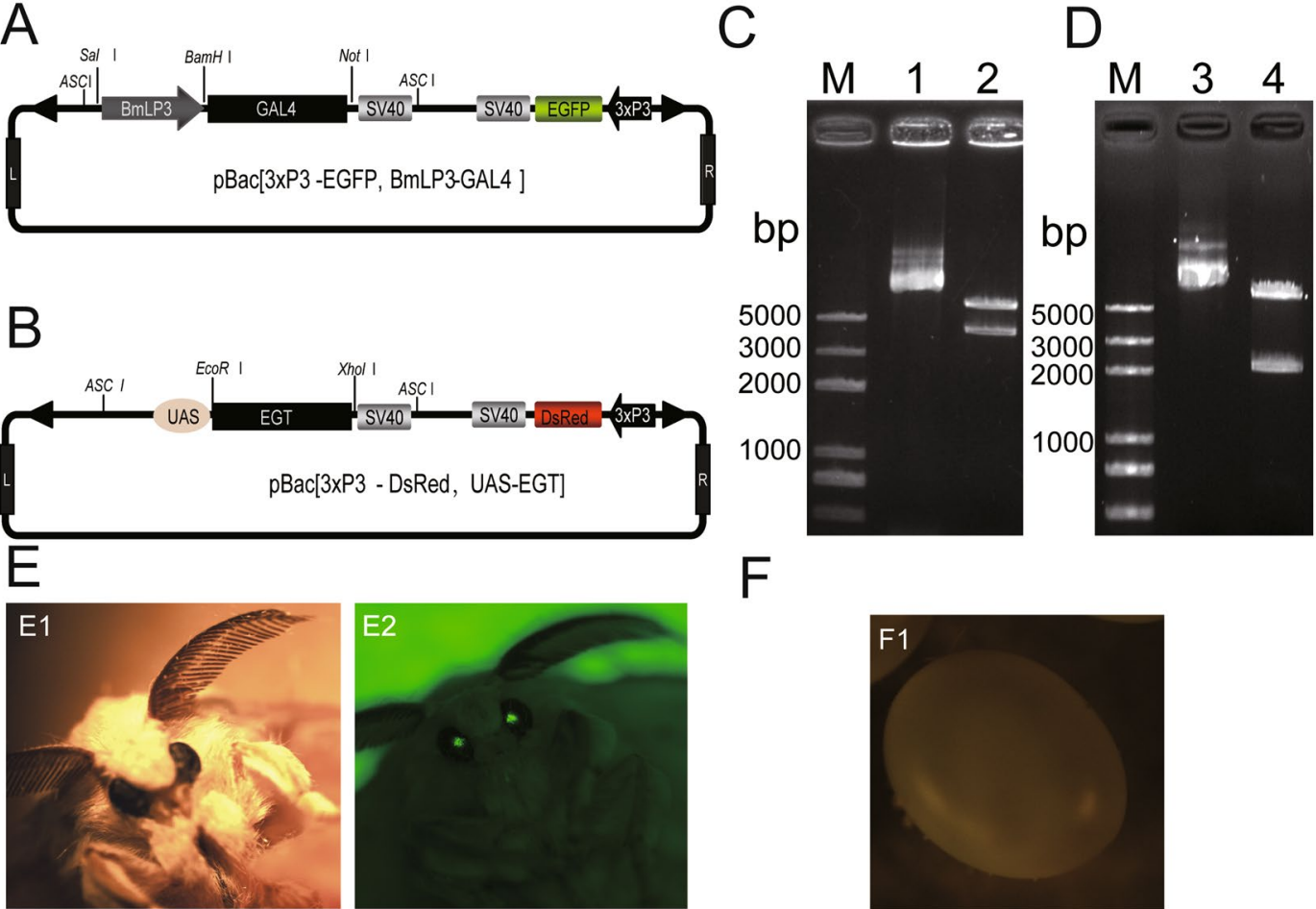

$\mathrm{F}$
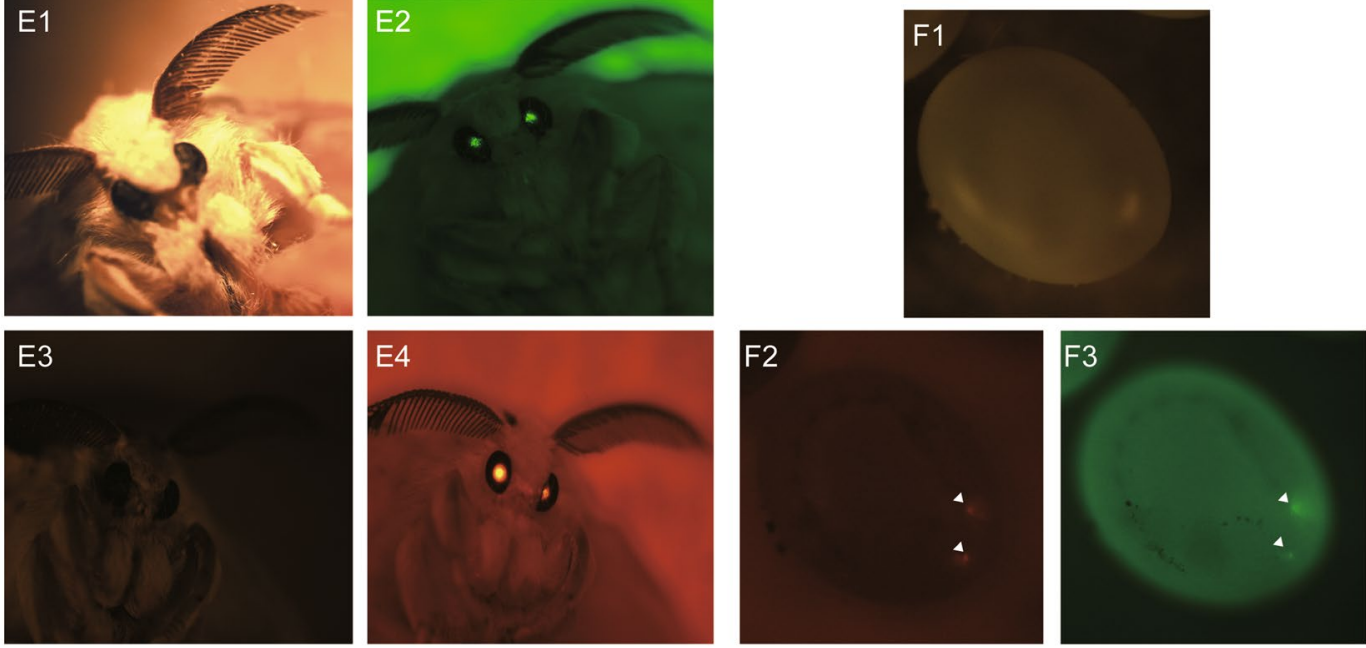

Figure 1. Isolation of transgenic silkworms. $(\mathbf{A}, \mathbf{B})$ Schematic illustration of the piggyBac $[3 \times \mathrm{P} 3-\mathrm{EGFP}, \mathrm{BmLP} 3$ Gal4] and piggyBac [ $3 \times$ P3-DsRed, UAS-EGT] vectors. $(\mathbf{C}, \mathbf{D})$ Digestion of the vectors with $A s c$ I yielded the expected band sizes. Lane 1/3: agarose gel electrophoresis detection of piggyBac [ $3 \times$ P3-EGFP, BmLP3-Gal4]/piggyBac [3 $\times$ P3-DsRed, UAS-EGT] vector plasmid; Lane 2/4: agarose gel electrophoresis detection of piggyBac [3 $\times$ P3-EGFP, BmLP3-Gal4]/piggyBac [ $3 \times$ P3-DsRed, UAS-EGT] vector plasmid after AscI digestion. BmLP3-Gal4-SV40 is about $4000 \mathrm{bp}$ and UAS-EGT-SV40 is about $2100 \mathrm{bp}$. (E) Isolation of generation G0 transgenic silkworms by detection of EGFP (Gal4 transgenic line) and DsRed (UAS transgenic line) expression in the compound eyes of moths. E1/E3: Gal4/ UAS transgenic moth under white light. E2/E4: Gal4/UAS transgenic moth under fluorescent light. (F) Screening of generation G7 hybrid offspring from Gal4 and UAS transgenic lines by detection of both EGFP and DsRed expression in the eggs. F1/2/3: transgenic eggs under white/green fluorescent/red fluorescent light.

gene of $B$. mori NPV in silkworm pupae was shown to prevent the emergence of silkworms and prolong the pupal stage $^{23}$. Thus, overexpressing EGT to reduce the content of active $20 \mathrm{E}$ in the haemolymph of late last-instar larvae can affect the progression of silkworm metamorphosis. We previously reported that a fragment of the B. mori $30 \mathrm{~K}$ lipoprotein (BmLP3) promoter expressed exclusively in the fat body of the late last-instar larval to early pupal stage could drive the expression of an exogenous protein with the same temporal and spatial distribution as endogenous LP $3^{24,25}$.

The GAL4/upstream activating sequence (UAS) expression system has been used in various model organisms including Arabidopsis thaliana ${ }^{26}$, Mus musculus ${ }^{27}$, Danio rerio ${ }^{28,29}$, and Drosophila melanogaster ${ }^{30}$. It has also been used to generate transgenic silkworms ${ }^{31,32}$ and expressing scorpion venom at the pupal stage ${ }^{33}$. Although EGT was shown to reduce the concentration of active $20 \mathrm{E}$ in silkworms, the toxic and lethal effects of EGT could affect productivity $^{23}$. In the present study, we used GAL4/UAS system to express EGT from the LP3 promoter in the silkworm fat body of last-instar larvae. The EGT protein secreted into the silkworm haemolymph maintained 20E at a low level, allowing us to investigate the role of $20 \mathrm{E}$ in the utilization of available nutrients for silk production.

\section{Results}

Generation of transgenic silkworms. We used EGT to reduce 20E content at the late last-instar larval stage by constructing two activator vectors, piggyBac [ $3 \times$ P3-EGFP, BmLP3-Gal4] (Fig. 1A) and piggyBac [3 $\times$ P3-DsRed, UAS-EGT] (Fig. 1B). Digestion of the plasmids with AscI yielded bands of the expected sizes (Fig. 1C,D). 
A

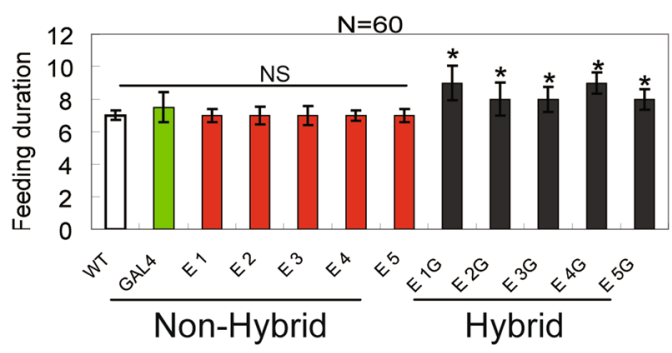

C
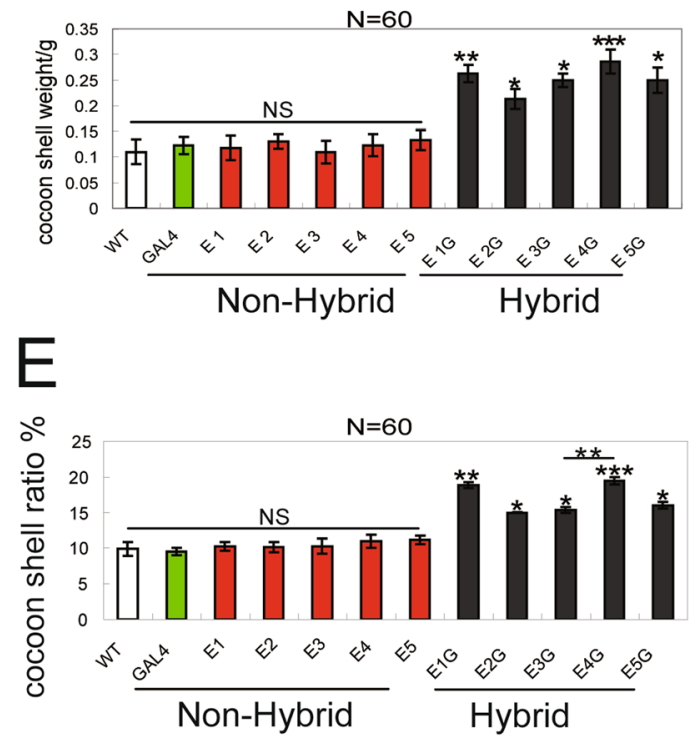

B

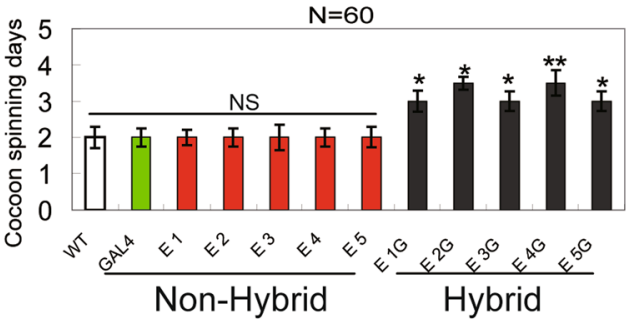

$\mathrm{D}$
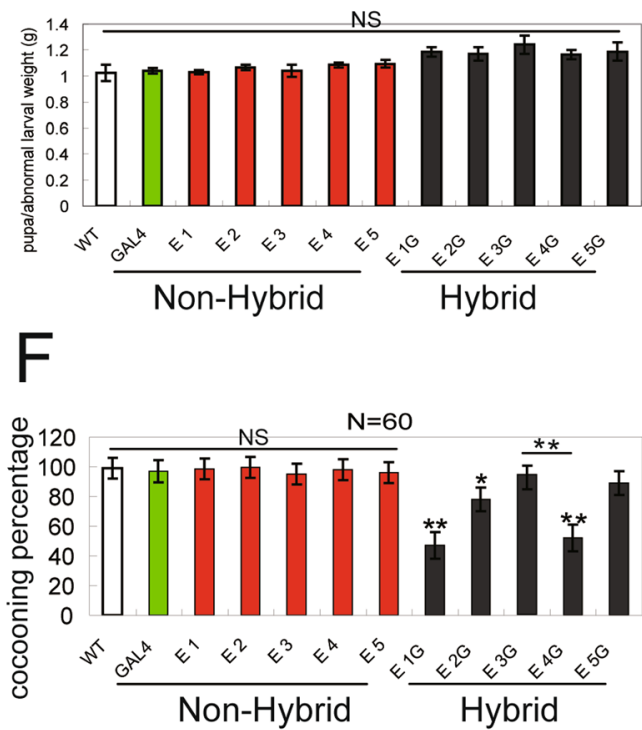

Figure 2. Last-instar larvae development. (A,B) Duration of feeding (A) and cocoon spinning (B) stages. (C) Cocoon shell weight. (D) Pupa/abnormal larval weight. (E) Cocoon shell ratio (in percentage). (F) Cocooning percentage.

After injecting the transgenic vectors into the eggs of silkworms, green/red fluorescence was detected in the compound eyes of generation G0 moths (Fig. 1E). Generation G7 of the BmLP3-Gal4 line was crossed with the G7 UAS-EGT transgenic line and the eggs laid by these moths showed both red and green fluorescence (Fig. 1F) indicating that the piggyBac vectors were inserted into the silkworm genome.

Last-instar larvae development and increased availability of nutrients in transgenic silkworms. We generated one Gal4 and five UAS-EGT transgenic lines that were reared on mulberry leaves up to generation G7, before intercrossing. The development and survival of hybrid offspring before the last-instar larval stage did not differ from the non-transgenic control (Fig. S1A/B). The number of feeding (Fig. 2A) and spinning (Fig. 2B) days and silk production (cocoon shell weight and ratio; Fig. 2C,E, respectively) of hybrid offspring at the last-instar larval stage were higher than those in non-transgenic or non-hybrid offspring. The cocooning percentage showed a decrease except for E3G and E5G lines (Fig. 2F), but the pupa/abnormal larvae weight showed no significant difference at cocoon spinning three days after (Fig. 2D). These results demonstrate that the extension of larval development leads to the increased amount of some silk proteins, although we did not observe any effect on silk fibroin.

EGT expression and function in transgenic silkworms. The hybrid offspring E3G cocoon shell ratio was increased (Fig. 2E) while cocooning percentage was unaltered (Fig. 2F). However, according to statistics of developmental time point in different strains, E3G silkworm larvae have a longer feeding and cocoon spinning stage and stay in pre-pupa that cannot pupate (Fig. 3A). In addition, the development of E3G was stopped between the larval and pupal stages, indicating that metamorphosis was blocked between these stages (Fig. 3B1). To confirm that the observed phenotypic effects resulted from the change of $20 \mathrm{E}$ content, enzyme-linked immunosorbent assay (ELISA) was performed to measure 20E level in last-instar larvae. The data revealed that the content of $20 \mathrm{E}$ in the haemolymph was lower in hybrid silkworms on days 7, 9, and 10 of the last-instar larval stage than in the control group. The control group $20 \mathrm{E}$ titers reached a peak at 5LD7, while 20E titer of E3G reached 

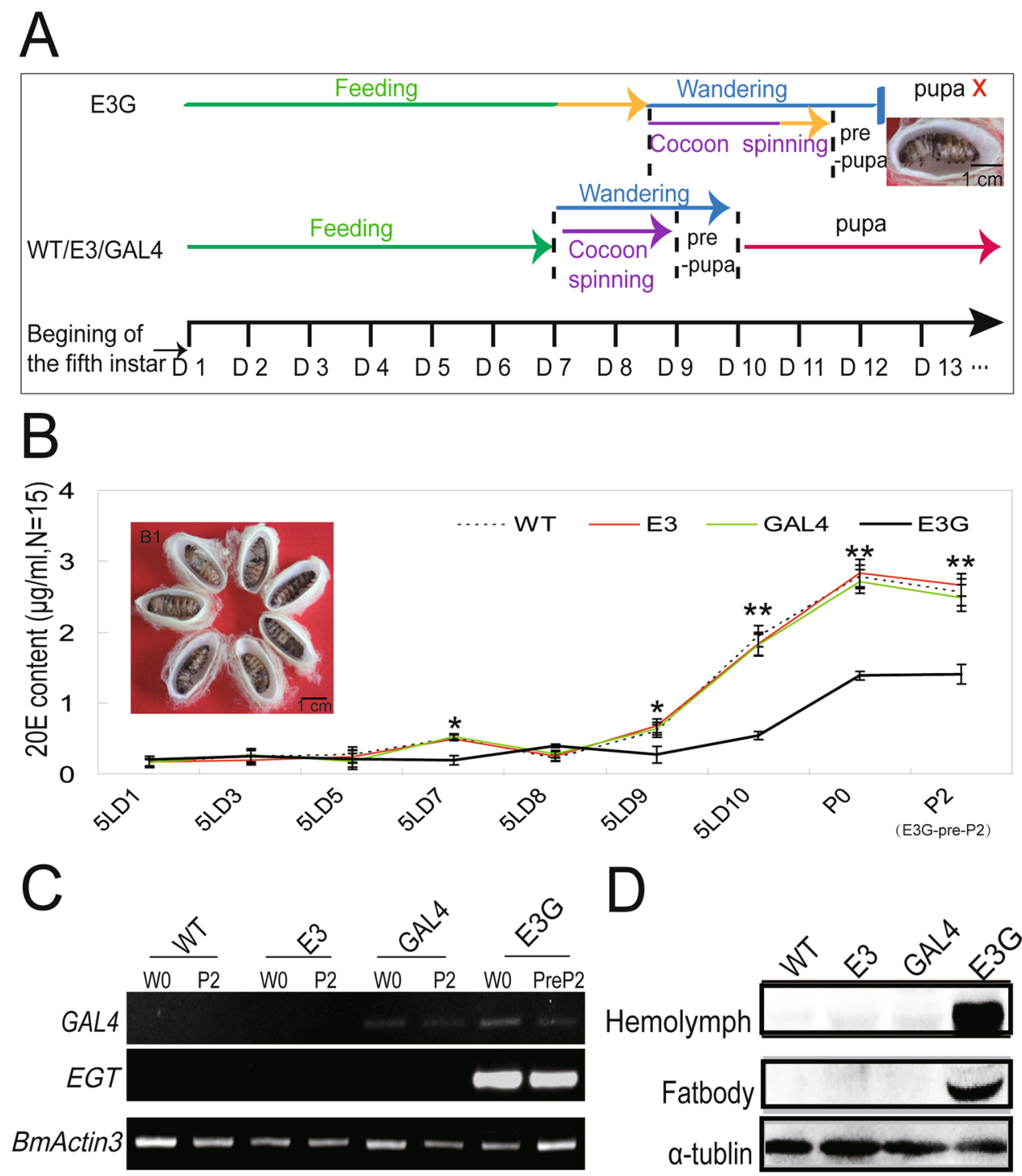

Figure 3. Phenotype, 20E content, and EGT expression of hybrid transgenic silkworm larvae (E3G). (A) Time point of developmental state among different silkworm strains. (B) Detection of 20E content in the haemolymph of last-instar larvae by ELISA. $\mathrm{B}_{1}$ Phenotype of E3G larvae on day 7 after wandering. 5LD1/3/5/7/8/9/10 indicate day 1/3/5/7/8/9/10 of fifth-instar larvae. (C) GAL4 and EGT mRNA levels in lastinstar larvae fat bodies at $0 \mathrm{~h}$ after wandering (W0) and day 2 of pupa/prepupa (P2/PreP2). (D) EGT protein level of last-instar larvae on day 3 after wandering.

a relatively small peak at 5LD8. So the appearance of the peak of 20E titers was delayed for about 1 day in E3G larvae (Fig. 3B), consistent with the prolonged feeding duration of the hybrid transgenic silkworm strains.

To further investigate if the decreased 20E content resulted from EGT expression, RNA was extracted from the fat body of last-instar larvae at feeding cessation (W0, spinning starts, larvae enter wandering stage) and day 2 of pupal stage (P2: non-hybrid silkworms, Pre-P2: hybrid silkworms-E3G). Reverse transcription PCR showed that Gal4 was expressed in GAL4 and E3G hybrid lines but not in E3, whereas EGT was strongly expressed in E3G only (Figs 3C and S3A). In addition, we were able to detect EGT protein in silkworm's haemolymph and fat body (Figs 3D and S3B). This indicated that the Gal4/UAS system was functional and that EGT over-expression in last-instar larvae reduced the $20 \mathrm{E}$ content in the haemolymph.

Cocoon composition in transgenic silkworms. Since the cocoons of the hybrid silkworm were bigger than that of the non-hybrid silkworms (Fig. 4A), we examined the characteristics of the cocoon by artificially reeling the silk from eight silkworms at the beginning of wandering. Optical microscope analysis revealed no differences in fibre diameter (Fig. 4B).

The cocoon is mainly composed of flavonoids, small molecules, and silk protein. We examined whether the increase in nutrient allocation to the cocoon affected its molecular composition and found that the solution obtained by dissolving hybrid transgenic silkworm cocoon was more yellow in colour than that of the control (Fig. 4C1). Two flavonoids were detected in the solution by high-performance liquid chromatography (HPLC; 
A

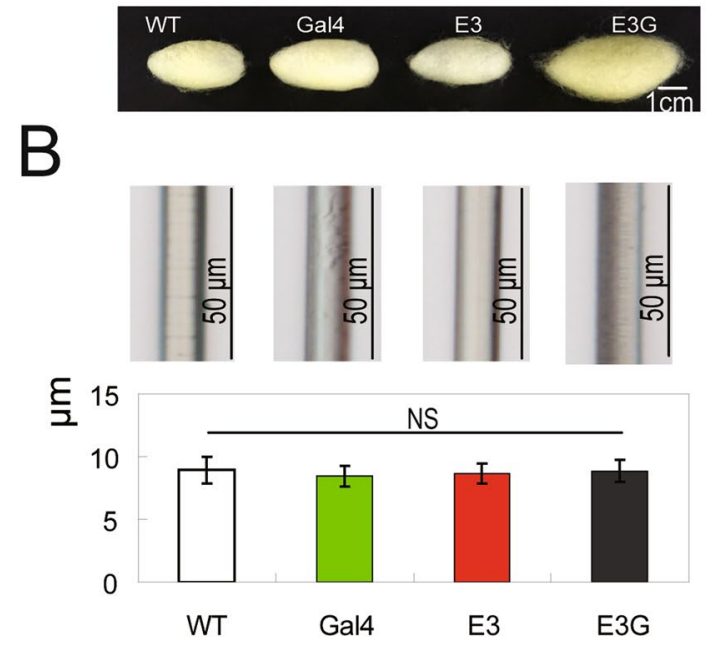

C

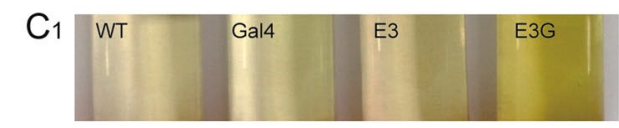

$\mathrm{C}_{2}$

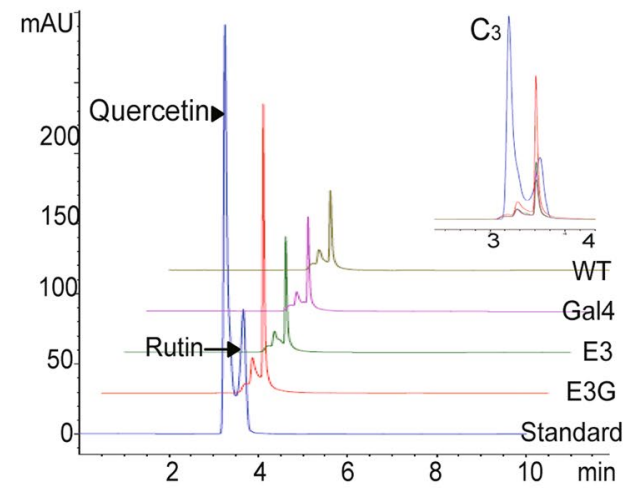

$\mathrm{D}$

$\mathrm{F}$
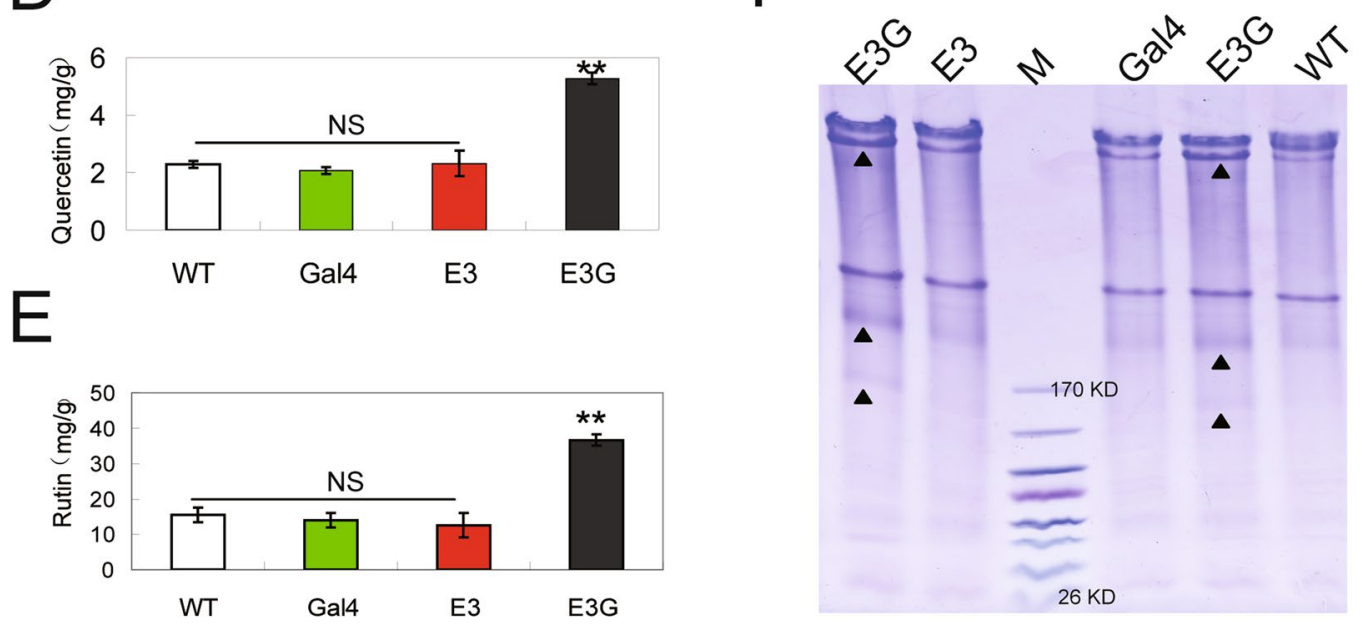

Figure 4. Detection of flavonoid and total sericin in the cocoon of transgenic silkworm. (A) Phenotype of cocoons. (B) Diameter of silk fibres produced by wandering-stage silkworms. (C) Detection of compounds in the cocoon by HPLC $\left(\mathrm{C}_{1}\right.$, flavonoid extract solution; $\mathrm{C}_{2}$, analysis of flavonoid extract solution by HPLC; $\mathrm{C}_{3}$, overlapping peaks in the chromatogram). (D,E) Quantitative analysis of quercetin/rutin content in cocoons by HPLC. (F) Analysis of total sericin content in cocoons by SDS-PAGE.

Fig. 4C2); these were identified as quercetin (Fig. 4D) and rutin (Fig. 4E). Further, to analyse the sericin content, the cocoon was cut into small pieces and degassed with an $8 \mathrm{M}$ urea solution at $100^{\circ} \mathrm{C}$ for 30 minutes. The supernatant was collected by centrifugation and used for sodium dodecyl sulphate polyacrylamide gel electrophoresis (SDS-PAGE). Results showed that sericin protein content (extracted from silkworm cocoons with the same weight in each group) was higher in the cocoon of hybrid transgenic silkworms than in the control group, especially at the position indicated by the black arrow in Fig. 4F. Thus, the decrease in 20E level resulted in flavonoid and sericin accumulation in the cocoon.

After removing sericin and small molecules, the cocoon contained only silk fibroin and its ratio (fibroin air-dried weight/cocoon shell weight $\times 100 \%$ ) in hybrid transgenic silkworm did not differ from that of control silkworm (Fig. 5A). Mechanical testing of silk fibroin revealed no differences in the stress-strain curves (Fig. 5B) or in average tenacity (Fig. 5C), breaking strain (Fig. 5D), toughness (Fig. 5E), or Young's modulus (Fig. 5F) between silk fibroin from non-transgenic and transgenic silkworms. Analysis of the secondary structure of silk fibroin by Fourier-transform infrared (FTIR) microspectroscopy (Fig. 6A) showed that the proportion of $\beta$-sheets (Fig. 6B), random coils (Fig. 6C), and $\alpha$-helices (Fig. 6D) were similar, whereas the proportion of $\beta$-turns (Fig. 6E) was higher in hybrid transgenic silkworm than in non-transgenic silkworm. These results demonstrate that a reduction in $20 \mathrm{E}$ titre at the last-instar larval stage had no impact on the mechanical properties of silk fibroin, although sericin protein accumulation was increased. 

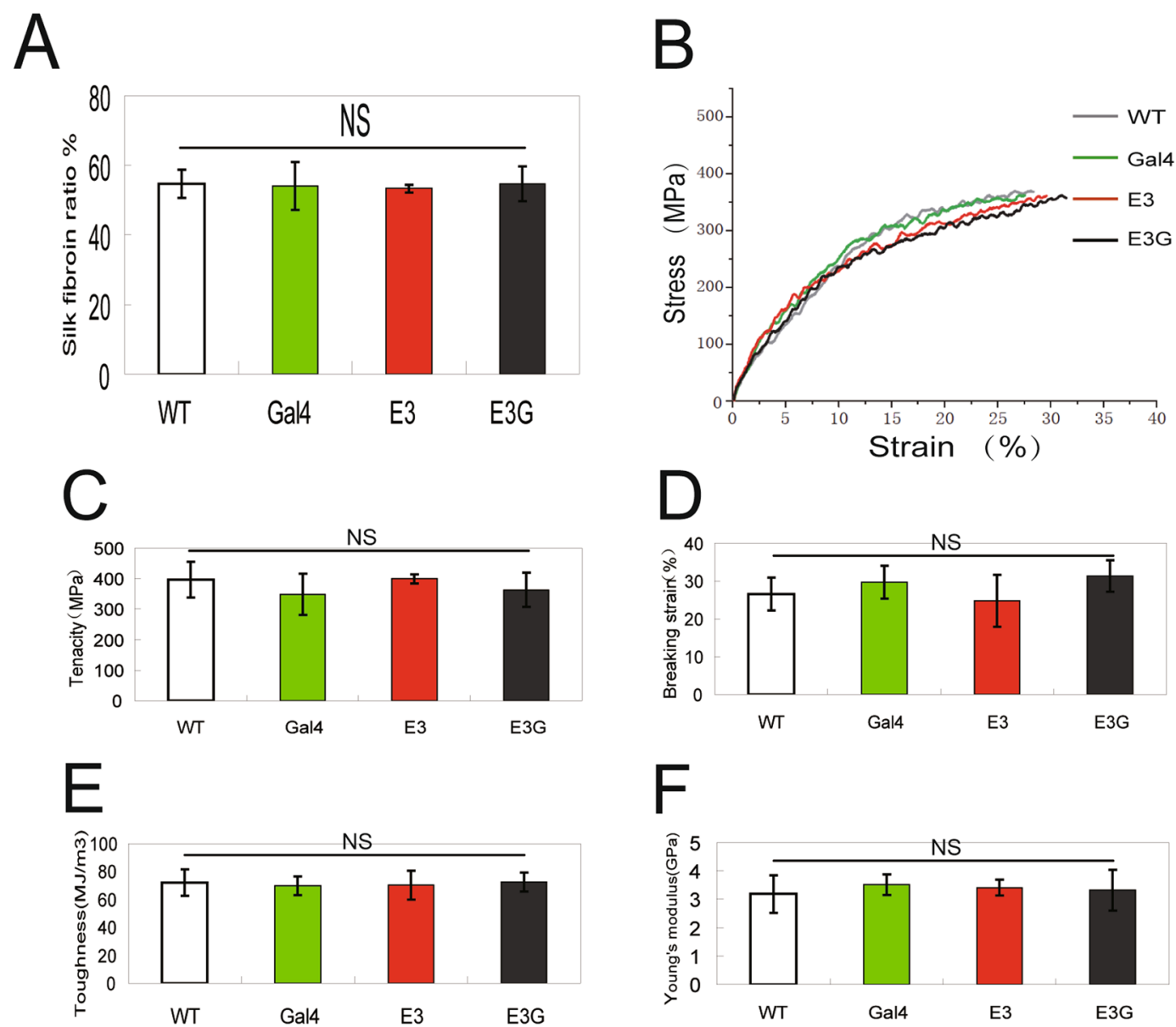

Figure 5. Mechanical properties of silk fibroin in transgenic silkworm. (A) Proportion of silk fibroin in the cocoon. (B) Stress-strain curves for silk fibroin. The curves represent an average of 30 experiments per group. (C-F) Tenacity (C), breaking strain (D), toughness (E), and Young's modulus (F) of silk fibroin obtained from wandering-stage silkworms.

Effect of exogenous 20E on last-instar larvae development and cocoon formation. Cocoon shell ratio (Fig. 2E) and cocooning percentage (Fig. 2F) varied among hybrid offspring. The cocoon shell ratio of E4G was higher than that of E3G (Fig. 2E) and they ceased developing at an earlier time point (Figs 3A1 and 7A); however, cocooning percentage in E4G (Fig. 2F) was only half that of E3G. On the other hand, both E3G and E4G hybrid transgenic fifth-instar larvae fed for about 1.5 days more than non-hybrid transgenic and non-transgenic silkworms (Fig. 7D). Male fifth-instar larvae showed normal cocoon spinning after they stopped eating, and the cocoon shell ratio of E3G male and E4G male was higher than that of non-hybrid transgenic E3 and E4. However, female E4G fifth-instar larvae showed almost no cocoon spinning until death, after they stopped feeding (Fig. 7E). Thus, the low cocooning percentage of $\mathrm{E} 4 \mathrm{G}$ was due to cocooning failure in $\mathrm{E} 4 \mathrm{G}$ females.

To examine the relationship between EGT expression level and 20E content with the development of E3G/ E4G, we compared EGT expression levels in E3G and E4G silkworms. The EGT transcript level in the fat body and EGT protein secretion into the haemolymph were both higher in E4G than in E3G (Fig. 8A). In addition, haemolymph $20 \mathrm{E}$ content was lower in $\mathrm{E} 4 \mathrm{G}$ than in E3G (Fig. 8B). Increased content of $20 \mathrm{E}$ after its administration through feeding (Fig. 8C) promoted cocoon spinning in female E4G silkworms (Fig. 8D) but decreased W0 larval weight (Fig. 8F) and cocoon shell ratio in males (Fig. 8G), although development was enhanced in both female (Fig. 8E) and male (Fig. 8H) silkworms. Thus, EGT over-expression in last-instar larvae decreases $20 \mathrm{E}$ content in the haemolymph, thereby leading to the extension of feeding and spinning periods and blocking pupation, which increases the availability of nutrients to the cocoon.

\section{Discussion}

It has been reported that juvenile hormone can inhibit $20 \mathrm{E}$ secretion during the early days of $B$. mori last (fifth)-instar larvae ${ }^{34}$, and extend the duration of feeding and silk yields ${ }^{35,36}$. Our study found that controlling $20 \mathrm{E}$ concentration via ectopic expression of EGT in the last larval instar can influence the duration of the larval feeding stage and cocoon shell ratio.

In hybrid transgenic silkworms, a significant reduction of $20 \mathrm{E}$ content was observed only from day 7 of fifth larval instar. However, no significant difference of $20 \mathrm{E}$ concentration was observed from the early fifth instar to the fifth day of the fifth larval instar (5LD5) between different silkworms strains. This might be due to the fact that 
A

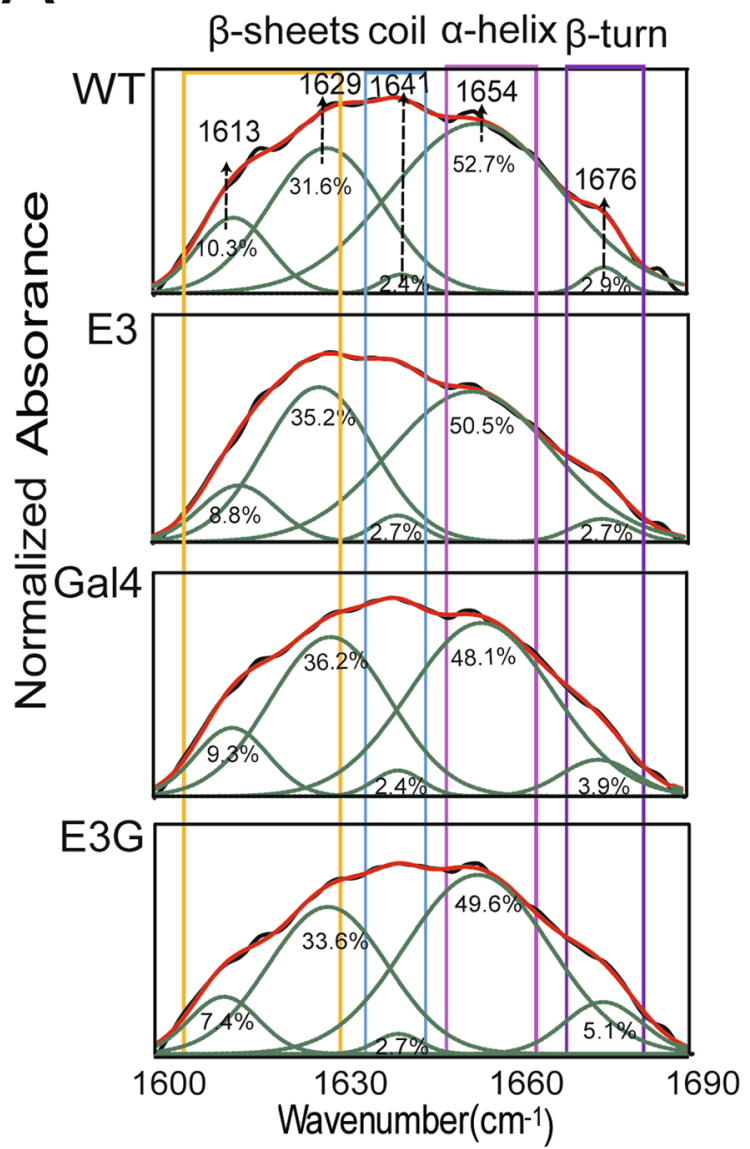

B

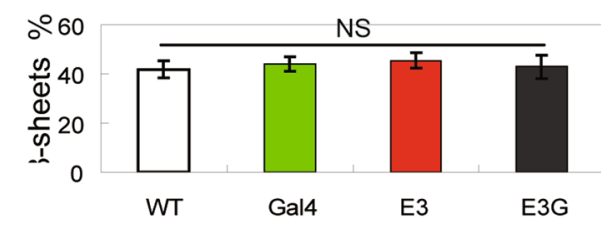

C

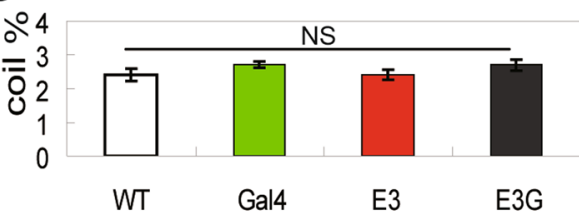

D
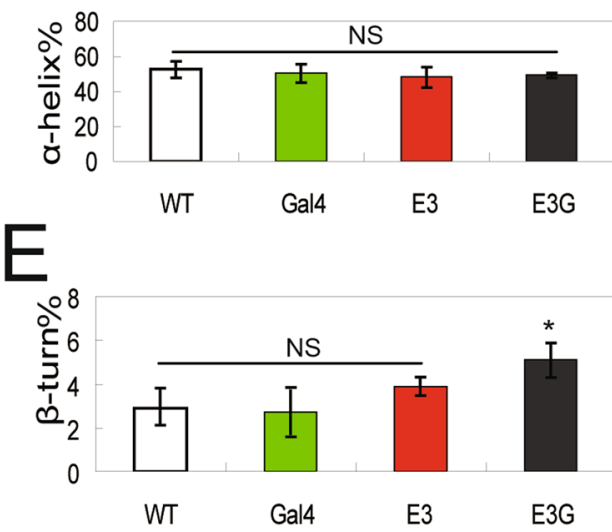

Figure 6. FTIR microspectroscopy analysis of silk fibroin secondary structure. (A) Curve fitting of the amide I region of silk fibroin in wandering-stage silkworms. (B-E) Proportion of $\beta$-sheets (B), random coils (C), $\alpha$-helices (D), and $\beta$-turns (E) in silk fibroin obtained from wandering-stage silkworms.

under the control of BmLP3, the larvae are expected to express the target gene only from day 4 of feeding in the fifth larval instar ${ }^{21}$. The silkworm last-instar larvae, after they stop feeding, empty their bowels and enter a period of maturation before starting to spin the cocoon. During this time, 20E titer shows a small increase ${ }^{10,37,38}$.

In the present study, compared with the control group, the peak of $20 \mathrm{E}$ titer in $\mathrm{E} 3 \mathrm{G}$ larvae was delayed for about 1 day (5LD7 for control group vs. 5LD8 for E3G, Fig. 3B). A prolongation of feeding duration by almost 1.5 days in hybrid transgenic larvae E3G thus might be related with the early expression of EGT to reduce the $20 \mathrm{E}$ content resulting in a delayed peak of $20 \mathrm{E}$ and a delay in the wandering and the spinning behaviour of the hybrid transgenic last-instar larvae. Further, E4G hybrid silkworm larvae, which have significantly lower content of $20 \mathrm{E}$ and much higher expression of EGT than E3G larvae ceased developing at an earlier time point than E3G larvae (Figs 3B1, 7A and 8A,B). In addition, females E4G could not form cocoon but E3G hybrid transgenic females could. However, female E4G is able to form normal cocoon after $20 \mathrm{E}$ treatment. The present study suggests thus the role of $20 \mathrm{E}$ in promoting cocoon spinning as $\mathrm{E} 4 \mathrm{G}$ females are able to form normal cocoons after 20E treatment.

E3G hybrid transgenic silkworms did not pupate normally after spinning and this could be due to the expression of EGT at the beginning of the cocooning and pre-pupal stage and a reduction of 20E content. Further, E4G larvae didn't enter into the normal pupal developmental program and this could be due to a higher expression of EGT and a severe reduction in 20E content in these larvae, as the pupal developmental program in these larvae was partly rescued after treatment with 20E. Moreover, E3G transgenic silkworms, expressing a relatively low level of EGT and an increased content of 20E than E4G, were unable to survive but showed some phenotypic characteristics of the pupae. Cocoon shell ratio was also lower in these hybrids than in silkworms with high EGT expression (E4G). In E3G larvae, as the expression of EGT is relatively low, the inactivation of 20E is also at a minimum. Thus, these larvae showed a relatively higher $20 \mathrm{E}$ content than E4G larvae. Wang et al. ${ }^{39}$ showed that the treatment of $20 \mathrm{E}$ decreases the consumption of food in silkworm larvae. Thus, a relatively greater level of $20 \mathrm{E}$ in E3G larvae might have decreased the consumption of food and the availability of nutrients towards cocoons formation, resulting in lower cocoon shell ratio when compared with E4G. Further, the treatment of hybrid transgenic male E4G silkworm larvae with 20E decreased the larval weight and cocoon shell ratio, confirming that the increase in the availability of nutrients to the cocoon and an increase in the cocoon shell weight in the hybrid transgenic silkworms is because of the EGT-induced reduction in active 20E content. 

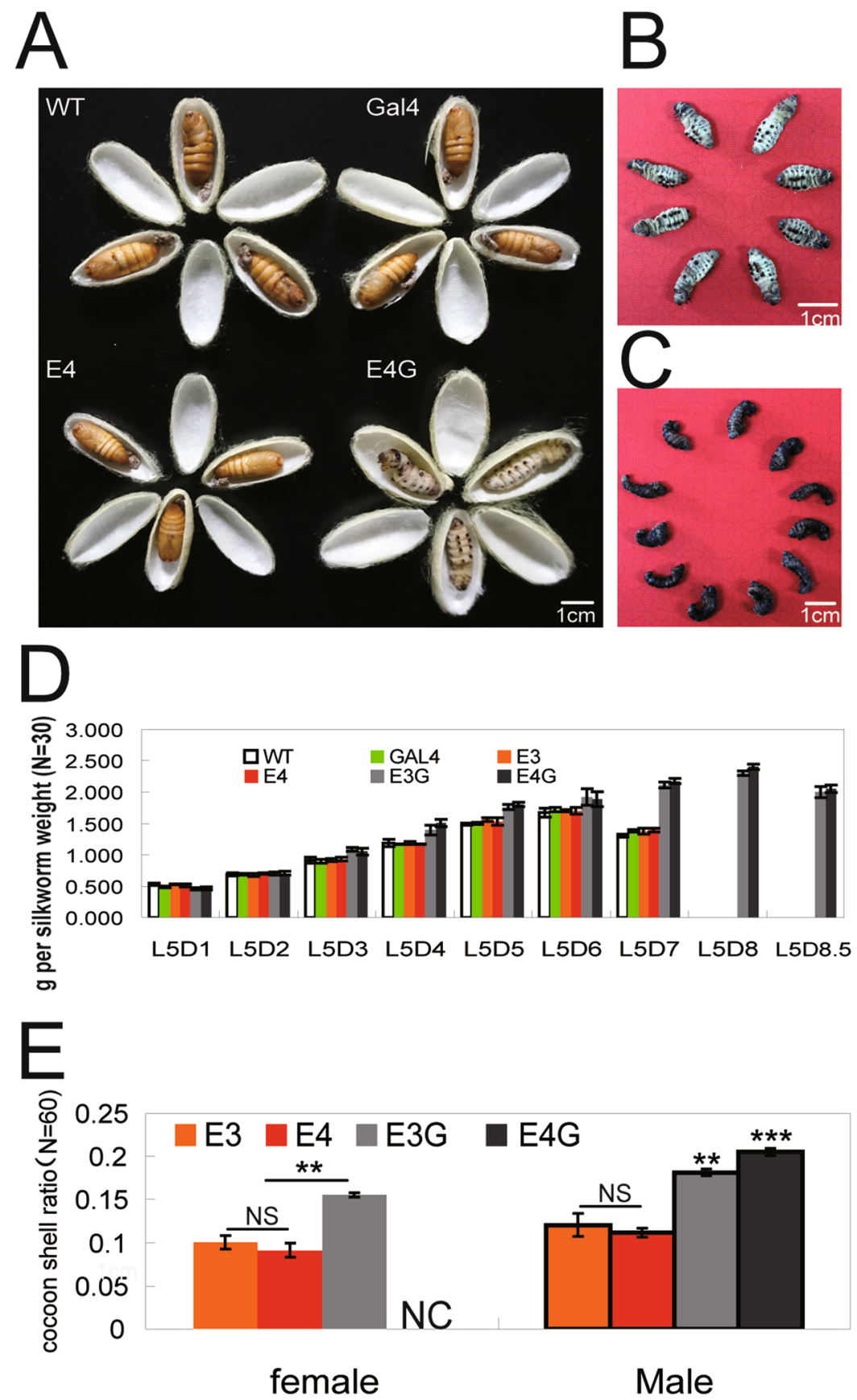

Figure 7. Last-instar larvae development in hybrid transgenic silkworm offspring. (A-C) Phenotype of transgenic hybrid silkworm E4G on days 7, 14, and 28 after wandering. (D) Weight and duration of feeding stage in last-instar larvae. (E) Cocoon shell ratios in male and female silkworms. NC indicates "No Cocoon".

Flavonoid content was increased in the cocoons of hybrid transgenic silkworms, and this is likely to be related to the homology between EGT and other endogenous enzymes (e.g. uridine diphosphate glucosyl transferase, NP-001135960.1) that catalyse flavonoid formation (Fig S2). We cannot exclude that the EGT would have an impact on some other enzymes, however, it seems more likely that the extension of the last larval instar causes the production of more flavonoids. Here, sericin content was increased in hybrid transgenic silkworms compared to non-transgenic silkworm; thus, the role of $20 \mathrm{E}$ in regulating the expression of silk protein genes warrants further investigation.

Silkworm is economically important due to its unique ability to spin silk. As such, a major concern in sericulture is to distribute most nutrients accumulated in larvae towards cocoon spinning to increase silk yield. Silkworm generated by traditional breeding methods has reached its upper limit for silk yield, and this can only be further improved using molecular biology-based approaches. Here, we developed a transgenic silkworm line in which EGT is expressed at the last-instar larval stage using the GAL4/UAS system. Overexpression of EGT decreased the content of active $20 \mathrm{E}$ in the haemolymph and blocked the development after cocoon spinning. Because pierced cocoons are unsuitable for reeling, pupae are killed by heating before eclosion, and silk is reeled 


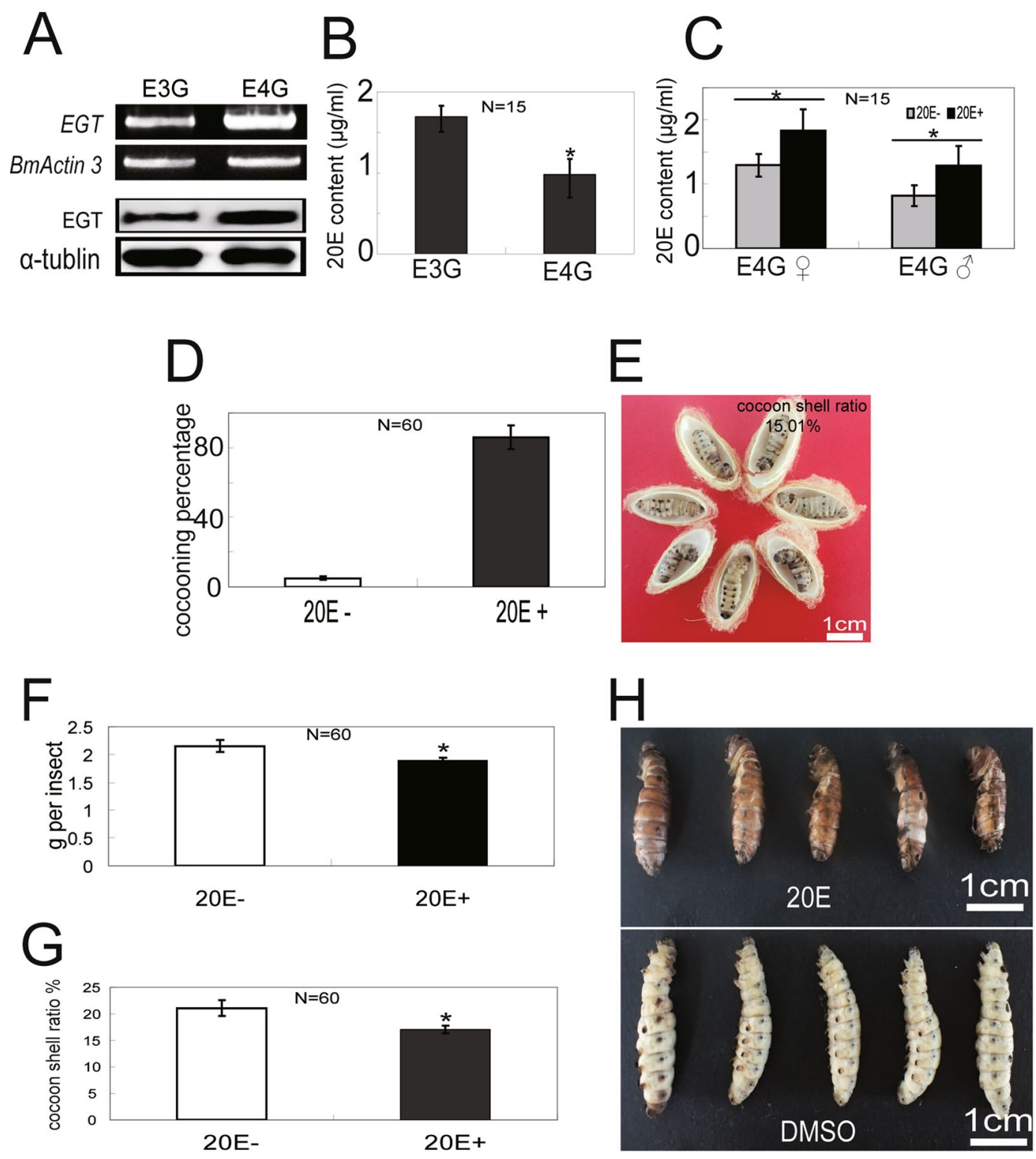

Figure 8. EGT expression and 20E content in E3G vs. E4G larvae and the effect of 20E administration on 20E content and larval development. (A,B) EGT expression level (A) and 20E content (B) in E3G and E4G lastinstar larvae $120 \mathrm{~h}$ after wandering. (C) Hemolymph $20 \mathrm{E}$ content in E4G female and male last-instar larvae 5 days after feeding on 20E-treated mulberry leaves. (D) E4G female cocooning percentage. (E) E4G female phenotype after 20E administration. (F) Larval weight of E4G W0 males. (G) Cocoon shell ratio of E4G males (in percentage). (H) E4G male phenotype after 20E administration.

from dried cocoons. However, heating/drying cocoons is a labour-intensive and energy-consuming process. In the present study, we generated transgenic silkworms that were developmentally arrested after spinning, which eliminates the labour required for fresh cocoon processing and drying and saves the energy required for drying. Thus, expression of EGT at the last-instar larval stage using the GAL4/UAS system is a reliable approach for industrial applications and saves the energy required for drying.

\section{Methods}

Construction of piggyBac transgenic vectors. Genomic DNA was isolated from B. mori strain Dazao and used to amplify the BmLP3 gene promoter fragment by PCR. The Gal4 gene was amplified from the pSL[A4GAL4] plasmid, which was constructed and stored in our laboratory. The following PCR profile was used to amplify target sequences: $95^{\circ} \mathrm{C}$ for $5 \mathrm{~min} ; 35$ cycles of $95^{\circ} \mathrm{C}$ for $30 \mathrm{~s}, 55^{\circ} \mathrm{C}$ for $30 \mathrm{~s}, 72^{\circ} \mathrm{C}$ for $1 \mathrm{~min}$; and $72^{\circ} \mathrm{C}$ for $10 \mathrm{~min}$. Amplicons were gel purified and cloned into the pMD19-T Simple plasmid (Takara Bio, Otsu, Japan). The recombinant plasmid was confirmed by sequencing. The EGT gene sequence containing an upstream activation sequence (UAS) with $A s c \mathrm{I}$ and $\mathrm{XhoI}$ sites was synthesized by Takara Bio Corporation and cloned into the pMD19-T Simple plasmid to generate pMD19-T [UAS-EGT]. 


\begin{tabular}{|c|c|c|c|c|c|}
\hline Gene & $\begin{array}{l}\text { Genbank } \\
\text { Accession No. }\end{array}$ & $5^{\prime}-3^{\prime}$ & Purpose & Sequence & Remark \\
\hline \multirow{2}{*}{ GAL4 } & \multirow{2}{*}{ AB186055.1 } & $\mathrm{F}$ & \multirow{6}{*}{ RT-PCR } & GCAATCCCATTACCGCATATC & \multirow{2}{*}{$431 \mathrm{bp}$} \\
\hline & & $\mathrm{R}$ & & AGGTTCGGACCGTTGCTACTGT & \\
\hline \multirow{2}{*}{ EGT } & \multirow{2}{*}{ AY048771.1 } & $\mathrm{F}$ & & CCAGCGTCCAGTATCTTG & \multirow{2}{*}{$649 \mathrm{bp}$} \\
\hline & & $\mathrm{R}$ & & CGACGACTGATGTCATGTC & \\
\hline \multirow{2}{*}{ BmActin3 } & \multirow{2}{*}{ AB701689.1 } & F & & AACACCCCGTCCTGCTCACTG & \multirow{2}{*}{$675 \mathrm{bp}$} \\
\hline & & $\mathrm{R}$ & & GGGCGAGACGTGTGATTTCCT & \\
\hline \multirow{2}{*}{$B m L P 3$} & \multirow{2}{*}{ FJ214661.1 } & F & \multirow{4}{*}{$\begin{array}{l}\text { For vector } \\
\text { construction in } \\
\text { transgenic larvae }\end{array}$} & GCgtcgacAGTATAGTTACAACGGCTGCCCC & \multirow{2}{*}{$1124 \mathrm{bp}$} \\
\hline & & $\mathrm{R}$ & & \begin{tabular}{|l|} 
CGggatccGGAGCGTCGAGTCCTGCAATATG \\
\end{tabular} & \\
\hline \multirow{2}{*}{ GAL4 } & \multirow{2}{*}{ AB186055.1 } & $\mathrm{F}$ & & CGggatccATGAAGCTACTGTCTTCTATCG & \multirow{2}{*}{$2646 \mathrm{bp}$} \\
\hline & & $\mathrm{R}$ & & TTgcggccgcTTACTCTTTTTTTGGGTTTGGTGG & \\
\hline
\end{tabular}

Table 1. Primers used in this study. Lower case letters indicate restriction sites.

The piggyBac vector containing $3 \times$ P3-EGFP can be used to screen transgenic silkworms. We constructed a piggyBac vector expressing the Gal4 gene under the control of the BmLP3 promoter as follows. The sequences were assembled into the intermediate plasmid pSL1180 (modified and stored in our laboratory) to generate a functional cassette, which was digested with AscI and ligated into piggyBac [ $3 \times \mathrm{P} 3$-EGFP] using T4 DNA ligase (New England Biolabs, Ipswich, MA, USA), yielding piggyBac [3 × P3-EGFP, Bm LP3-Gal4].

Similarly, a piggyBac vector with $3 \times \mathrm{P} 3$-DsRed as a screening marker was prepared as follows. The AscI-XhoI fragment of the PCR fragment from pMD19-T [UAS-EGT] was inserted into the AscI-XhoI site of pSL1180 to generate the intermediate plasmid pSL1180 [UAS-EGT]. The cassette was excised and inserted into piggyBac $[3 \times \mathrm{P} 3$-EGFP] to generate piggyBac [3 $\times$ P3-DsRed, UAS-EGT].

Generation of transgenic silkworms. Transgenic silkworms were generated as previously described ${ }^{40}$ using the plasmids piggyBac [3 × P3-EGFP, Bm LP3-Gal4] and piggyBac [3 3 P3-DsRed, UAS-EGT]. Generation G0 moths were screened for the expression of EGFP or DsRed in their compound eyes under an epifluorescence stereomicroscope (Olympus, Tokyo, Japan). The EGFP-/DsRed-positive individuals were mated with siblings and the offspring were raised for seven generations and maintained at the State Key Laboratory of Silkworm Genome Biology of Southwest University, Chongqing, China.

Hybrid descendants of Gal4 and UAS transgenic silkworms. Generations G7 of BmLP3-Gal4 and UAS-EGT transgenic lines were crossed, and the hybrid offspring were reared on mulberry leaves. Data on feeding and wandering days of last-instar larvae, cocoon shell ratio (cocoon shell weight/whole cocoon weight $\times 100$ ), cocooning percentage (total number of cocoons/total number of matured larvae $\times 100$ ), and survival rate were recorded.

FTIR microspectroscopy and analysis of the mechanical properties of silk fibers. At the beginning of cocoon spinning, eight silkworms were randomly selected and silk fibres were reeled off at $40 \mathrm{rpm}$; the first $500 \mathrm{~m}$ were discarded, and $50 \mathrm{~m}$ of fibre were degummed twice in a $0.5 \% \mathrm{NaHCO}_{3}\left(\mathrm{wt} \%\right.$ ) solution at $100{ }^{\circ} \mathrm{C}$ for $30 \mathrm{~min}$. The silk was washed and air-dried at room temperature and cut to lengths of $16 \mathrm{~mm}$; the diameter of 30 randomly selected segments was measured by optical microscope (JEOL, Tokyo, Japan) $(n=3)$. The FTIR microspectroscopy procedures and the analysis of mechanical properties were carried out as previously described ${ }^{40}$ (Breaking strain describes the plastic properties of materials. Material fracture after total deformation, $\Delta \mathrm{L}$ percentage of the original gauge length $(\mathrm{L})$ ratio; Young's modulus: describes the material elastically deformed. The larger the value, the more rigid is the material).

Detection of flavonoids in sericin by HPLC. Eight cocoons were randomly selected and cut into pieces; $0.5 \mathrm{~g}$ of cocoon tissue was added to $200 \mathrm{ml} 0.5 \% \mathrm{NaHCO}_{3}$ (wt \%) solution at $100^{\circ} \mathrm{C}$ for $30 \mathrm{~min}$, and then cooled to room temperature before centrifugation at $10,000 \times \mathrm{g}$ at $25^{\circ} \mathrm{C}$ for $30 \mathrm{~min}$. The flavonoid content of the supernatant was determined by HPLC using a C18 column $(250 \mathrm{~mm} \times 4.6 \mathrm{~mm}$ inner diameter, $5-\mu \mathrm{m}$ particles $)$ at $35^{\circ} \mathrm{C}$. The mobile phase was acetonitrile and $0.1 \%$ formic acid $(75: 25, \mathrm{v} / \mathrm{v})$; injection volume was $10 \mu \mathrm{l}$; flow rate was $0.4 \mathrm{ml} / \mathrm{min}$; and detection wavelength was $330 \mathrm{~nm}$.

Detection of EGT expression in hybrid offspring of Gal4 and UAS transgenic silkworms. Reverse transcription PCR. Fat bodies were dissected from silkworms ( $\mathrm{n}=15$ per group) and placed in sterile polyethylene tubes. Total RNA was extracted using TRIzol reagent (Invitrogen, Carlsbad, CA, USA) and Moloney murine leukaemia virus reverse transcriptase (Invitrogen) was used to generate first-strand cDNA according to the manufacturer's instructions. PCR amplification was carried under the following conditions: $95^{\circ} \mathrm{C}$ for $30 \mathrm{~s}$, and 25 cycles of $95^{\circ} \mathrm{C}$ for $30 \mathrm{~s}$ and $60^{\circ} \mathrm{C}$ for $30 \mathrm{~s}$. BmActin 3 was used as a control. Primer sequences are listed in Table 1 . Three independent replicates were analysed.

SDS-PAGE and western blotting. Total protein was quantified using a bicinchoninic reagent kit (Invitrogen) and $40 \mu \mathrm{g}$ per sample were resolved by SDS-PAGE on $8 \%$ and $3-12 \%(\mathrm{w} / \mathrm{v})$ polyacrylamide gradient gels for detection of EGT and sericin, respectively, according to the method of Laemmli ${ }^{41}$. Proteins were 
electrophoretically transferred to a polyvinylidene difluoride membrane (Roche Diagnostics, Indianapolis, IN, USA) that was probed with anti-EGT antibody (EGT protein was expressed in prokaryotic cells and provided to immunized rabbit to obtain EGT-polyclonal antibody) diluted 1:5000 in Tris-buffered saline with Tween 20 ( $10 \mathrm{mmol} / \mathrm{l} \mathrm{Tris-HCl}$, [pH 7.5], $150 \mathrm{mmol} / \mathrm{l} \mathrm{NaCl}$, and $0.05 \%$ Tween 20$)$ containing $1 \%$ (w/v) bovine serum albumin. Horseradish peroxidase-conjugated goat anti-rabbit IgG (Beyotime Institute of Biotechnology, Shanghai, China) diluted 1: 10,000 in blocking buffer was used as the secondary antibody. Immunocomplexes were detected by enhanced chemiluminescence (Thermo Fisher Scientific, Waltham, MA, USA) and blots were imaged using a Clinx ChemiScope 3400 Mini (Science Instruments Co., Beijing, China).

Treatment of larvae with 20E. On day 7 of the last-instar larval stage, 20E was sprayed on mulberry leaves fed to larvae at a dose of $1 \mu \mathrm{g} / \mathrm{day} / \mathrm{g}$ body weight until they stopped eating; dimethylsulfoxide was used as a control. Each group contained 60 larvae. To determine the amount of $20 \mathrm{E}$ sprayed onto mulberry leaves, silkworms within each group were weighed. Briefly, if the total weight of the 60 larvae was $60 \mathrm{~g}$, we would dissolve $60 \mu \mathrm{g} 20 \mathrm{E}$ in $500 \mu \mathrm{l}$ sterile water, and then spray this solution onto appropriate amount of mulberry leaves. These leaves were mixed with $20 \mathrm{E}$ and they were fed to silkworms when the solution on the leaves was dry. When silkworms finished these treated mulberry leaves, they were fed on normal mulberry leaves.

Measurement of 20E level. Haemolymph was taken from the silkworm removing a proleg collected in a sterile polyethylene tube, and then centrifuged at $5000 \times \mathrm{g}$ for $10 \mathrm{~min}$ at $4{ }^{\circ} \mathrm{C}$. To prevent melanisation of haemolymph, phenylthiourea was added to the supernatant at a final concentration of $2 \mathrm{mmol} / \mathrm{l}$. Hemolymph samples from 15 silkworms were pooled. The concentration of $20 \mathrm{E}$ was measured as previously described ${ }^{42}$.

Statistical analysis. All values are the mean \pm SD, differences between groups were analysed with Student's t-tests, considering $\mathrm{P}<0.05$ as the significance threshold. Single asterisk and double asterisks presented in figures indicate significant differences between treated and control groups at $\mathrm{P}<0.05$ and $\mathrm{P}<0.01$, respectively, and NS indicates no significant difference $(\mathrm{p}>0.05)$.

\section{References}

1. Xia, Q. et al. A draft sequence for the genome of the domesticated silkworm (Bombyx mori). Science 306, 1937-1940, https://doi. org/10.1126/science.1102210 (2004).

2. Mita, K. et al. The genome sequence of silkworm, Bombyx mori. DNA Res 11, 27-35, https://doi.org/10.1093/dnares/11.1.27 (2004).

3. Hamamoto, H., Tonoike, A., Narushima, K., Horie, R. \& Sekimizu, K. Silkworm as a model animal to evaluate drug candidate toxicity and metabolism. Comp Biochem Phys C 149, 334-339, https://doi.org/10.1016/j.cbpc.2008.08.008 (2009).

4. Matsumoto, Y., Sumiya, E., Sugita, T. \& Sekimizu, K. An invertebrate hyperglycemic model for the identification of anti-diabetic drugs. PLoS One 6, e18292, https://doi.org/10.1371/journal.pone.0018292 (2011).

5. Tansil, N. C. et al. The use of molecular fluorescent markers to monitor absorption and distribution of xenobiotics in a silkworm model. Biomaterials 32, 9576-9583, https://doi.org/10.1016/j.biomaterials.2011.08.081 (2011).

6. Yuan, H. X., Xu, X., Sima, Y. H. \& Xu, S. Q. Reproductive toxicity effects of 4-nonylphenol with known endocrine disrupting effects and induction of vitellogenin gene expression in silkworm, Bombyx mori. Chemosphere 93, 263-268, https://doi.org/10.1016/j. chemosphere.2013.04.075 (2013).

7. Kiguchi, K. Time-Table for the Development of the Silkworm, Bombyx-Mori. Jarq-Jpn Agr Res Q 17, 41-46 (1983).

8. Sakurai, S. Temporal Organization of Endocrine Events Underlying Larval Pupal Metamorphosis in the Silkworm, Bombyx-Mori. J Insect Physiol 30, 657-664, https://doi.org/10.1016/0022-1910(84)90051-9 (1984).

9. Calvez, B., Hirn, M. \& De Reggi, M. Ecdysone changes in the haemolymph to two silkworms (Bombyx mori and Philosamia cynthia) during larval and pupal development. FEBS Lett 72, 57-61, https://doi.org/10.1016/0014-5793(76)80898-8 (1976).

10. Mizoguchi, A., Ohashi, Y., Hosoda, K., Ishibashi, J. \& Kataoka, H. Developmental profile of the changes in the prothoracicotropic hormone titer in hemolymph of the silkworm Bombyx mori: correlation with ecdysteroid secretion. Insect Biochem Molec 31, 349-358, https://doi.org/10.1016/S0965-1748(00)00127-2 (2001).

11. Matsui, H., Kakei, M., Iwami, M. \& Sakurai, S. Hormonal regulation of the death commitment in programmed cell death of the silkworm anterior silk glands. J Insect Physiol 58, 1575-1581, https://doi.org/10.1016/j.jinsphys.2012.09.012 (2012).

12. Terashima, J., Yasuhara, N., Iwami, M. \& Sakurai, S. Programmed cell death triggered by insect steroid hormone, 20-hydroxyecdysone, in the anterior silk gland of the silkworm, Bombyx mori. Dev Genes Evol 210, 545-558, https://doi.org/10.1007/s004270050345 (2000).

13. Tsuzuki, S., Iwami, M. \& Sakurai, S. Ecdysteroid-inducible genes in the programmed cell death during insect metamorphosis. Insect Biochem Mol Biol 31, 321-331, S0965174800001247 (2001).

14. Uhlirova, M. et al. Use of Sindbis virus-mediated RNA interference to demonstrate a conserved role of Broad-Complex in insect metamorphosis. P Natl Acad Sci USA 100, 15607-15612, https://doi.org/10.1073/pnas.2136837100 (2003).

15. Reza, A. M. S. et al. Hormonal control of a metamorphosis-specific transcriptional factor Broad-Complex in silkworm. Comp Biochem Phys B 139, 753-761, https://doi.org/10.1016/j.cbpc.2004.09.009 (2004).

16. Yan, Q. S., Ouyang, X. G., Li, C. B. \& Pang, Y. Insect baculovirus-encoded ecdysteroid UDP-glucosyltransferases. Prog Biochem Biophys 26, 241-242 (1999).

17. Ahrens, C. H. et al. The sequence of the Orgyia pseudotsugata multinucleocapsid nuclear polyhedrosis virus genome. Virology 229, 381-399, https://doi.org/10.1006/viro.1997.8448 (1997).

18. Chaturvedi, P., Misra, P. \& Tuli, R. Sterol Glycosyltransferases-The Enzymes That Modify Sterols. Appl Biochem Biotech 165, 47-68, https://doi.org/10.1007/s12010-011-9232-0 (2011).

19. Evans, O. P. \& O’Reilly, D. R. Purification and kinetic analysis of a baculovirus ecdysteroid UDP-glucosyltransferase. Biochem J 332(Pt 3), 807-808 (1998).

20. Oreilly, D. R. \& Miller, L. K. A Baculovirus Blocks Insect Molting by Producing Ecdysteroid Udp-Glucosyl Transferase. Science 245, 1110-1112, https://doi.org/10.1126/science.2505387 (1989).

21. Shikata, M. et al. The ecdysteroid UDP-glucosyltransferase gene of Autographa californica nucleopolyhedrovirus alters the moulting and metamorphosis of a non-target insect, the silkworm, Bombyx mori (Lepidoptera, Bombycidae). J Gen Virol 79(Pt 6), 1547-1551, https://doi.org/10.1099/0022-1317-79-6-1547 (1998).

22. Hoover, K. et al. A gene for an extended phenotype. Science 333, 1401, https://doi.org/10.1126/science.1209199 (2011).

23. Zhang, X. et al. Effects of egt gene transfer on the development of Bombyx mori. Gene 491, 272-277, https://doi.org/10.1016/j. gene.2011.09.026 (2012). 
24. Deng, D. J. et al. The promoter of Bmlp3 gene can direct fat body-specific expression in the transgenic silkworm, Bombyx mori. Transgenic Research 22, 1055-1063, https://doi.org/10.1007/s11248-013-9705-8 (2013).

25. Xu, H. et al. Identification of a functional element in the promoter of the silkworm (Bombyx mori) fat body-specific gene Bmlp3. Gene 546, 129-134, https://doi.org/10.1016/j.gene.2014.05.038 (2014).

26. Waki, T. et al. A GAL4-based targeted activation tagging system in Arabidopsis thaliana. Plant J 73, 357-367, https://doi. org/10.1111/tpj.12049 (2013).

27. Sauter, K. A. et al. The MacBlue Binary Transgene (csf1r-gal4VP16/UAS-ECFP) Provides a Novel Marker for Visualisation of Subsets of Monocytes, Macrophages and Dendritic Cells and Responsiveness to CSF1 Administration. PLoS One 9, https://doi.org/10.1371/ journal.pone.0105429 (2014).

28. Liu, S. \& Leach, S. D. Screening Pancreatic Oncogenes in Zebrafish Using the Gal4/UAS System. Method Cell Biol 105, 367-381, https://doi.org/10.1016/B978-0-12-381320-6.00015-1 (2011).

29. Kawakami, K. et al. Gal4 Driver Transgenic Zebrafish: Powerful Tools to Study Developmental Biology, Organogenesis, and Neuroscience. Adv Genet 95, 65-87, https://doi.org/10.1016/bs.adgen.2016.04.002 (2016).

30. Fischer, J. A., Giniger, E., Maniatis, T. \& Ptashne, M. GAL4 activates transcription in Drosophila. Nature 332, 853-856, https://doi. org/10.1038/332853a0 (1988).

31. Imamura, M. et al. Targeted gene expression using the GAL4/UAS system in the silkworm Bombyx mori. Genetics 165, 1329-1340 (2003).

32. Xu, H. \& O'Brochta, D. A. Advanced technologies for genetically manipulating the silkworm Bombyx mori, a model Lepidopteran insect. Proc Biol Sci 282, https://doi.org/10.1098/rspb.2015.0487 (2015).

33. Zhang, H. K. et al. Effects of BmKIT 3 R gene transfer on pupal development of Bombyx mori Linnaeus using a Gal4/UAS binary transgenic system. J Agric Food Chem 60, 3173-3179, https://doi.org/10.1021/jf300172u (2012).

34. Sakurai, S., Okuda, M. \& Ohtaki, T. Juvenile hormone inhibits ecdysone secretion and responsiveness to prothoracicotropic hormone in prothoracic glands of Bombyx mori. Gen Comp Endocrinol 75, 222-230, https://doi.org/10.1016/0016-6480(89)900749 (1989).

35. Akai, H., Kiguchi, K. \& Mori, K. Increased accumulation of silk protein accompanies KJH-induced prolongation of larval life in Bombyx mori L. Applied Entomology and Zoology 6, 218-220 (1971).

36. Akai, H., Kimura, K., Kiuchi, M. \& Shibukawa, A. Increase of silk production by repeated treatments with a juvenile hormone analogue. J Seric Sci Jpn 54, 297-299 (1985).

37. Satake, S., Kaya, M. \& Sakurai, S. Hemolymph ecdysteroid titer and ecdysteroid-dependent developmental events in the last-larval stadium of the silkworm, Bombyx mori: role of low ecdysteroid titer in larval-pupal metamorphosis and a reappraisal of the head critical period. J Insect Physiol 44, 867-881, S0022191098000754 (1998).

38. Gu, S. H. \& Chow, Y. S. Role of Low Ecdysteroid Levels in the Early Last Larval Instar of Bombyx-Mori. Experientia 49, 806-809, https://doi.org/10.1007/Bf01923554 (1993).

39. Wang, S. et al. 20-hydroxyecdysone Reduces Insect Food Consumption Resulting in Fat Body Lipolysis During Molting and Pupation. Journal of Molecular Cell Biology 3, 128-138, https://doi.org/10.1093/jmcb/mjq006 (2010).

40. Wang, X. et al. Modifying the Mechanical Properties of Silk Fiber by Genetically Disrupting the Ionic Environment for Silk Formation. Biomacromolecules 16, 3119-3125, https://doi.org/10.1021/acs.biomac.5b00724 (2015).

41. Laemmli, U. K. Cleavage of structural proteins during the assembly of the head of bacteriophage T4. Nature 227, 680-685 (1970).

42. Meng, M. et al. The Homeodomain Transcription Factors Antennapedia and POU-M2 Regulate the Transcription of the Steroidogenic Enzyme Gene Phantom in the Silkworm. Journal of Biological Chemistry 290, 24438-24452, https://doi.org/10.1074/ jbc.M115.651810 (2015).

\section{Acknowledgements}

We thank Dr. Xin Wang for the excellent assistance with the mechanical properties experiments of silk fibres. This work was supported by the State Key Program of National Natural Science of China (Grant No. 31530071), the National Natural Science Foundation of China (Grant No. 31472154) and the Fundamental Research Funds for the Central Universities (Grant No. XDJK2017B006).

\section{Author Contributions}

G.S. performed the experiments, collected and analysed the data, and wrote the paper; J.W., Y.W., H.L., H.Z., S.M., C.P. and Y.L. participated in the experiments and collected data; Q.X. designed the experiments and analysed the data. All authors reviewed the manuscript.

\section{Additional Information}

Supplementary information accompanies this paper at https://doi.org/10.1038/s41598-018-36261-y.

Competing Interests: The authors declare no competing interests.

Publisher's note: Springer Nature remains neutral with regard to jurisdictional claims in published maps and institutional affiliations.

(c) (i) Open Access This article is licensed under a Creative Commons Attribution 4.0 International

License, which permits use, sharing, adaptation, distribution and reproduction in any medium or format, as long as you give appropriate credit to the original author(s) and the source, provide a link to the Creative Commons license, and indicate if changes were made. The images or other third party material in this article are included in the article's Creative Commons license, unless indicated otherwise in a credit line to the material. If material is not included in the article's Creative Commons license and your intended use is not permitted by statutory regulation or exceeds the permitted use, you will need to obtain permission directly from the copyright holder. To view a copy of this license, visit http://creativecommons.org/licenses/by/4.0/.

(c) The Author(s) 2018 